\title{
1 Fire Spread Predictions: Sweeping uncertainty under the rug
}

3 Akli Benali ${ }^{\mathrm{a},}{ }^{*}$, Ana C.L. Sá ${ }^{\mathrm{a}}$, Ana R. Ervilha ${ }^{\mathrm{b}}$, Ricardo M. Trigo ${ }^{\mathrm{c}}$, Paulo M. Fernandes ${ }^{\mathrm{d}}$, José

$4 \quad$ M.C. Pereira ${ }^{a}$

5

6

$7 \quad{ }^{a}$ Centro de Estudos Florestais, Instituto Superior de Agronomia, Universidade de Lisboa, Tapada

8 da Ajuda, Lisboa, Portugal.

9 b LAETA, IDMEC, Instituto Superior Técnico, Universidade Lisboa, Departamento de

10 Engenharia Mecânica, LASEF, Av. Rovisco Pais, 1, Lisboa, Portugal

$11{ }^{\mathrm{c}}$ Instituto Dom Luís, Faculdade de Ciências da Universidade de Lisboa, Campo Grande Edifício

12 C8, Piso 3, Lisboa, Portugal

13 d Centro de Investigação e de Tecnologias Agro-Ambientais e Biológicas, Universidade de Trás-

14 os-Montes e Alto Douro, Quinta de Prados, Vila Real, Portugal

$16 *$ Corresponding author.

17 Email: aklibenali@gmail.com $(\mathrm{AB})$

20 Keywords: satellite; FARSITE; MODIS; hotspots; likelihood; Generalized Likelihood 21 Uncertainty Estimation (GLUE) 


\section{Abstract}

24 Predicting fire spread and behavior correctly is crucial to minimize the dramatic 25 consequences of wildfires. However, our capability of accurately predicting fire spread is still 26 very limited, undermining the utility of such simulations to support decision-making. Improving 27 fire spread predictions for fire management purposes, by using higher quality input data or 28 enhanced models, can be expensive, unfeasible or even impossible. Fire managers would benefit 29 from fast and inexpensive ways of improving their decision-making. In the present work, we 30 focus on i) understanding if fire spread predictions can be improved through model parameter 31 calibration based on information collected from a set of large historical wildfires in Portugal; and 32 ii) understanding to what extent decreasing parametric uncertainty can counterbalance the impact 33 of input data uncertainty. Our results obtained with the Fire Area Simulator (FARSITE) 34 modeling system show that fire spread predictions can be continuously improved by 'learning' 35 from past wildfires. The uncertainty contained in the major input variables (wind speed and 36 direction, ignition location and fuel models) can be 'swept under the rug' through the use of 37 more appropriate parameter sets. The proposed framework has a large potential to improve future 38 fire spread predictions, increasing their reliability and usefulness to support fire management and 39 decision making processes, thus potentially reducing the negative impacts of wildfires. 


\section{Introduction}

Wildfires are a disruptive phenomenon with important environment and socio-economic impacts. Accurately predicting and anticipating fire spread and behavior is crucial to minimize dramatic consequences. For this purpose, fire spread models have been widely used to support fire management decisions, such as in real-time fire behavior prediction (Kochanski et al. 2013), anticipated fire risk assessment (Calkin et al. 2011), fire suppression preparedness (Sneeuwjagt and Peet 1985) and fire and fuel hazard mitigation resulting from planned fuel treatments (Ager et al. 2010).

The capability of accurately predicting fire spread is still very limited, and associated uncertainties strongly undermine the utility of such predictions for decision-making (Alexander and Cruz 2013a). Modeling fire behavior is uncertain mainly due to imperfect scientific knowledge regarding the mechanisms driving fire spread, model applicability and its inherent limitations, input data quality, natural variability, and parametric uncertainty (Albini 1976; Alexander and Cruz 2013b; Ervilha et al. 2017; Liu et al. 2015; Refsgaard et al. 2007; Thompson and Calkin 2011). In a general sense, the lack of knowledge (epistemic uncertainty), rather than simple random variability, can be responsible for important prediction errors (Beven and Binley 2014). For instance, it has been shown that errors in input data can lead to large prediction errors (Albini 1976; Anderson et al. 2007; Bachmann and Allgöwer 2002; Benali et al. 2016a).

There is a certain inability of the current fire-research modeling community to completely take into account the strong limitations imposed by the pervasive levels of uncertainties. This is of paramount relevance, as fire spread simulations will only be deemed useful if they can provide reliable information to fire managers. Understanding how simulations can be improved is, therefore, a critical research task that can contribute to mitigate negative downstream consequences. For example, in an operational context, anticipating correctly where and when a location will burn, and the corresponding level of confidence, is important to define suppression strategies (Pinto et al. 2016). On the other hand, in a pre-operational context, improving fire spread predictions can, for example, render more reliable assessments of fire risk and improve fuel management decisions (Ager et al. 2010; Salis et al. 2013).

Currently, there are many fire spread models available that range from empirical to physically-based (Sullivan 2009a, b, c). Each option has advantages and disadvantages that 
depend on several aspects, such as computational and data demand, costs, accuracy, complexity, among others (Papadopoulos and Pavlidou 2011). Among these, the Fire Area Simulator (FARSITE) modeling system (Finney 2004) has been widely used to simulate the spread and behavior of individual fires. Its accuracy, easiness to use, along with its moderate complexity, data size demands and computation times, have been recognized by many authors (Arca et al. 2007; Papadopoulos and Pavlidou 2011; Salis et al. 2016; Sullivan 2009c). FARSITE, along with several other fire modeling systems, uses the Rothermel semi-empirical fire spread model (Rothermel 1972) to predict rate of spread (ROS) at any given spread direction of a surface fire. It is based on topographic, weather and vegetation information. The latter is based on fuel models that consist of a numerical description of the structure and composition of surface organic matter capable of flaming combustion (Anderson 1982). Fuel models are composed by several parameters describing the fuel complex, with different impacts on the expected fire behavior (Ervilha et al. 2017; Liu et al. 2015)

Fire spread predictions can be improved in a number of ways, namely by i) increasing scientific knowledge driving fire behavior and spread mechanisms; ii) developing more accurate and reliable models; iii) using higher quality input data; and iv) model calibration. However, we have different levels to improve these "four horses of apocalypse" that hamper fire-spread model results. Improving data, models and scientific knowledge, may involve challenging tasks that are too expensive and time consuming. Additionally, the complexity of models can significantly undermine their application by fire managers. Consequently, the characteristics of these options rarely coincide with the demands and requirements of fire managers for short-term and inexpensive improvements of fire spread predictions.

Within this context, model calibration can be a relatively inexpensive, fast and simple way of improving fire spread predictions, and consequently, decision-making. Several fire modeling systems have enclosed in their model structure parameters (i.e. the empirical values constant throughout the simulations) that can be adjusted with the objective of improving the agreement between estimated and observed fire spread and behavior (Cruz and Alexander 2010; Finney 2004; Mandel et al. 2014). Among these, the calibration of fuel model parameters has been often done with significant improvements to fire spread prediction accuracy (Ascoli et al. 2015; Cai et al. 2014; Cruz and Fernandes 2008; Rothermel and Rinehart 1983; Salis et al. 2016). Nevertheless, the large uncertainties associated with the lack of detailed and accurate 
information required for fuel mapping at large spatial scales (Keane and Reeves 2012), as well as the spatial variability within each mapping unit (Hilton et al. 2015), can significantly jeopardize the utility of fuel model calibration for prediction improvement.

Alternatively, Duguy et al. (2007) used FARSITE to reproduce the fire spread patterns of an historical event by tuning the ROS adjustment factors, scalars that multiplied by the estimated ROS and that do not affect other fire behavior outputs. Contrary to several parameters that are not easily accessible to the average fire model user for model calibration, these empirical factors are used to rapidly adjust the fire spread rate based on the expected or observed fire behavior for each individual fuel model (Finney 2004; Rothermel and Rinehart 1983). Despite this effort, the potential improvement of fire spread predictions that result from tuning such empirical parameters remains largely unknown. In particular, it is still unknown if this simple calibration approach can be applied to other wildfires to effectively reduce prediction errors, or if they are mostly case-specific and have little effectiveness in improving predictions of subsequent wildfires.

We explore whether the calibration of the empirical ROS adjustment factors of FARSITE can be a simple, fast and inexpensive way of improving the consequent fire spread predictions. We do not consider the uncertainties associated with fuel model parameters that have been studied elsewhere (Ascoli et al. 2015; Bachmann and Allgöwer 2002; Ervilha et al. 2017; Liu et al. 2015). The impact of data uncertainty is taken into account based on preceding work (see Benali et al. 2016a). Investigating other sources of uncertainty is outside the scope of the work, however, the readers are referred to Thompson and Calkin (2011) and Webley et al. (2016) for further information. Here, we propose to i) quantify how fire spread predictions can be improved through model parameter calibration based on information collected from historical large wildfires; and ii) understand to what extent decreasing parametric uncertainty can counterbalance the impact of input data uncertainty. For this purpose, the fire spread predictions are evaluated using satellite active fire data for seven large historical wildfires in Portugal that occurred between 2003 and 2005. Understanding and quantifying the sources of prediction error, or producing the best possible predictions, is beyond the scope of the work, as we focus on the relative improvements made by calibrating the fire modeling system. 


\section{Data and Methods}

\subsection{Fire Spread Simulations}

We selected seven very large wildfires that occurred in Portugal between 2003 and 2005. Each wildfire burned between $\sim 13,700$ ha and 40,000 ha and lasted for several days. These historical case studies were above the $99^{\text {th }}$ percentile of fire size distribution considering all the wildfires that occurred between 1975 and 2013 in mainland Portugal (Sá et al. 2017). The location, burned area perimeter, fire name and respective acronym are displayed in S1 Fig. 1, along with their characteristics shown in S1 Table 1. The burned area perimeters of all case studies were extracted from the Landsat-derived Portuguese fire atlas (Oliveira et al. 2012). The ignition locations, start and end date of the case studies were defined using satellite active fire data (Benali et al. 2016b).

We used FARSITE to simulate the fire spread patterns of the case studies. FARSITE uses distinct models for surface fire spread (Rothermel 1972), crown fire transition (van Wagner 1977), and crown fire spread (Rothermel 1991). We used FARSITE 4 command line version to simulate surface fire, with a landscape cell-size of $100 \mathrm{~m}$ and an hourly time step. Spotting, crown fires and fire suppression were not simulated due to their stochastic nature and the lack of information, respectively.

FARSITE requires a comprehensive set of landscape and weather variables. Slope and aspect were derived from the digital elevation data acquired from the NASA Shuttle Radar Topography Mission (SRTM) (Farr et al. 2007). Fuel maps were produced by reclassifying the Corine Land Cover (Bossard et al. 2000) classes into fire behavior fuel models as per the Northern Forest Fire Laboratory (NFFL; Anderson 1982). The reclassification key and the correspondent fuel maps are shown in S2. Initial dead fuel moisture contents were set to $6 \%, 7 \%$ and $8 \%$, for 1-hr, 10-hr and 100-hr time-lag classes, respectively, based on Scott and Burgan (2005). Live fuel moisture contents were set to $60 \%$ and $90 \%$, for herbaceous and woody components, respectively. Canopy cover density was extracted from the Moderate Resolution Imaging Spectroradiometer (MODIS) Vegetation Continuous Fields (VCF) product (DiMiceli et al. 2011). Weather variables were derived from a high-resolution dataset based on PSU/NCAR mesoscale model (MM5) simulations (Lorente-Plazas et al. 2015) and used as hourly data at $10 \mathrm{~km}$ resolution. A 
comprehensive list of the required input variables to model fire spread using FARSITE is provided in S3 Table 1.

Using the set of input variables described above and setting the adjustment factors to one (i.e. no adjustment) we performed the reference fire spread simulations for the seven historic case studies. In sections 2.3 and 2.4 we describe how the uncertainty in the ROS adjustment factors and input variables was integrated in the fire spread simulations.

\subsection{Evaluation of fire spread simulations}

Evaluating fire spread simulations against static burned area perimeters ignores the spatiotemporal patterns of fire spread, not effectively contributing to improve predictions (Cui and Perera 2010; Duff et al. 2013; Filippi et al. 2014). Alternatively, the MODIS active fire product (MCD14ML) uses thermal data to identify the location of fires burning at the time of overpass with a nominal spatial resolution of $1 \mathrm{~km}^{2}$ (Giglio et al. 2003). MODIS is aboard two satellites, resulting in four distinct acquisition periods per day on average: Terra data are acquired during day and nighttime at around 10:30 - 12:00 a.m./p.m. local time, respectively, and Aqua data at around 1:00 - 3:00 a.m./p.m, respectively. MODIS active fire data are specially suited to monitor large and long-lasting wildfires (Anderson et al. 2009; Hawbaker et al. 2008; Parks 2014; Veraverbeke et al. 2014).

Recently, Sá et al. (2017) proposed evaluating the accuracy of fire spread simulations using satellite active fire data. The evaluation scheme is based on quantifying the spatial discrepancy (hereafter, $S p D$ ) between fire spread simulations and fire growth observed by satellite thermal acquisitions. The $S p D$ is defined by the authors as the minimum Euclidean distance (in $\mathrm{km}$ ) between a satellite active fire pixel and the nearest simulated cell burning at the time of satellite overpass. Due to the uncertainty in the sub-pixel location of the fire front, the minimum Euclidean distance between all the possible sub-pixel locations within the MODIS active fire pixel and the closest simulated burned pixel was calculated. We made some modifications to the evaluation scheme proposed by Sá et al. (2017) to use only the most outward satellite active fires in the $S p D$ calculation. Details are provided in $\mathrm{S} 2$. The spatial discrepancy was assumed to be an indicator of prediction accuracy, such that a low discrepancy was interpreted as a close match between satellite-observed and simulated fire growth. 


\subsection{Uncertainty Quantification}

In this section we describe the quantification of the uncertainty associated with fire spread modeling inputs, specifically with variables and parameters. The uncertainty associated with the most relevant input variables has been estimated by Benali et al. (2016a). Here, we provide only a brief overview of the latter work (section 2.3.1). Quantification of the uncertainty associated with model parameters was focused in the ROS adjustment factors and is described in section 2.3.2. Here after, model parameters refer to the ROS adjustment factors unless stated otherwise. Finally, we describe how the impact of the uncertainty associated with input variables and parameters on the output fire spread predictions was assessed (section 2.3.3).

\subsubsection{Model Variables}

The uncertainty associated with the input variables was estimated previously, using different methods and independent data sources, along with its impact on fire spread predictions (Benali et al. 2016a). Here, based on the previous study results, we focused on the variables for which the uncertainty had a larger impact on the prediction accuracy: wind speed and direction, ignition location, and fuel model assignment.

We sampled 100 values from the uncertainty histograms of wind speed and direction, and ignition location variables (see Figure 4c-e in Benali et al. 2016a). For the wind-related variables, uncertainty was propagated by adding the sampled uncertainty value to the reference value, and generating the correspondent fire spread simulation. For the ignition location, we generated random ignition points within the burned area perimeter with a distance to the reference location equal to the sampled uncertainty value. The uncertainty associated with the assignment of NFFL fuel models based on CLC land cover classes was calculated using a confusion matrix (see Table 2 in Benali et al. 2016a). A total of 100 combinations of land coverfuel model assignments were defined and a simulation was performed for each combination. The uncertainties were propagated through the fire model for each case study independently, one variable at a time.

\subsubsection{Model Parameters: The ROS adjustment factors}

To the best of our knowledge, there is no data available regarding the 'true' distribution of the ROS adjustment factors. We estimated their probability distribution through inverse 
modeling following the Generalized Likelihood Uncertainty Estimation (GLUE) methodology (Beven and Binley 1992). The underlying rationale is that models are fraught with uncertainties, hence a "true" parameter set that provides an "optimal" fit to the observed data does not exist. Instead, different parameter sets can produce equivalent and equally acceptable predictions, leading to equifinality. The likelihood of a parameter set being an adequate system simulator was assessed and used to provide an estimate of the associated uncertainty. The estimation of the uncertainty associated with the ROS adjustment factors using GLUE required the following steps:

i) A large number of fire spread simulations with randomly assigned parameter sets;

ii) The estimation of the likelihood of each parameter set;

iii) The use of the likelihood as weights to estimate uncertainty;

iv) The update of the likelihood weights using new data.

Steps i), ii) and iii) are described in the current section, while step iv) is described in section 2.4 .

The GLUE methodology requires an appropriate definition of the prior parameter distributions. In the current work, a parameter set is a vector with ROS adjustment factor values for each NFFL fuel model. Considering the lack of a priori information regarding the distributions of the ROS adjustment factors, a wide parameter range was defined (0.33 to 3 ) and Uniform distributions were used. The parameter range corresponds to a 3 fold decrease and increase in the estimated ROS, respectively. For each case study, only the fuel models with at least $2.5 \%$ of areal coverage and summing up at least $95 \%$ of the total simulation area were considered as representative. We randomly assigned parameter values to the most representative fuel models and then performed one fire spread simulation per parameter set, for a total of 500 simulations. Parameter sets were exactly the same for all case studies.

GLUE requires that an appropriate likelihood measure is defined and calculated for each parameter set. The term likelihood is used in a general sense, as the possibility that a given parameter set leads to an agreement between model predictions and satellite active fires. The likelihood was formally defined as: 


$$
L_{i, j}=\left(\frac{1}{S p D_{i, j}}\right)^{2}
$$

where $L_{i, j}$ is the likelihood and the $S p D_{i, j}$ is the simulation-satellite spatial discrepancy of the $i$-th parameter set for the $j$-th case study (see section 2.2). A large spatial discrepancy means that a given parameter set has a low (or even null) likelihood of being a system simulator. The inverse relationship. All $S p D_{i, j}$ below $0.5 \mathrm{~km}$ were truncated to avoid numerical problems (i.e. division by zero) and pronounced extremes in the likelihood function.

The likelihoods were rescaled for each case study so that the sum of all values equaled 1, yielding the probability density function of the parameter sets. The uncertainty associated with the ROS adjustment factors was estimated by using the previously calculated likelihood values to weight the correspondent simulation. A higher likelihood was translated into a larger weight of a given parameter set and its correspondent simulation, and vice versa. The weights were used to quantify the impact of uncertainty in the model outputs, i.e. predictive uncertainty, explained in detail in section 2.3.3.

\subsubsection{Predictive uncertainty}

The impact of the uncertainty associated with input variables and parameters on the fire spread predictions was assessed by calculating the spatial discrepancy ratio and the burn probability. The former provides information regarding the impact of uncertainty on simulation accuracy and the latter on the estimated fire growth spatial patterns. The spatial discrepancy ratio was defined as (Benali et al. 2016a):

$$
S p D_{\text {ratio }_{i, j}}(\%)=\frac{S p D_{i, j}-S p D_{R E F_{j}}}{S p D_{R E F_{j}}} \times 100
$$

where $S p D_{\text {ratioi,j }}$ is the spatial discrepancy ratio of the $i$-th parameter set for the $j$-th case study and $S p D_{R E F j}$ is the spatial discrepancy for the reference simulation for the $j$-th case study. A positive ratio means that propagating uncertainty leads to a larger satellite-simulated discrepancy when compared with the reference simulation, thus to less accurate fire spread predictions. The likelihoods $\left(L_{i, j}\right)$ were used to weight each $S p D_{\text {ratioi } j}$, yielding a distribution of values for each $j$-th case study. 
Some authors have proposed to integrate uncertainty into fire spread predictions using probabilistic approaches (Cruz 2010; Finney et al. 2011; Pinto et al. 2016). Instead of a deterministic estimation of fire spread, the probability of a given pixel burning (hereafter, burn probability) was estimated by performing multiple simulations integrating uncertainty. We estimated the burn probability by using the likelihoods $\left(L_{i, j}\right)$ to weight the corresponding fire growth simulation. The latter was reclassified to 1 and 0 , i.e. burned and unburned, for the corresponding simulation period. Burn probability maps were reclassified into six discrete probability classes based on Pollack (2005; see S5 Table 1). Additionally, we estimated the burn probability resulting from each main input variable uncertainty and compared with that resulting from the integration of the ROS adjustment factors uncertainty, by calculating the absolute burn probability difference between both. For the input variable uncertainty, each simulation had the same weight.

\subsection{The impact of new data on predictive uncertainty and accuracy}

We investigated how integrating new data regarding the likelihood of the parameter sets influenced the predictive uncertainty and the accuracy of fire spread predictions. Using the GLUE methodology and Bayes' theorem, the likelihood of the parameter sets can be updated (i.e posterior likelihood) combining prior with new likelihood estimates (Beven and Binley 1992):

$$
L_{p}(\Theta \mid y)=L_{y}(\Theta \mid y) L_{o}(\Theta)
$$

where $L_{o}(\Theta)$ is the prior likelihood distribution, $L_{p}(\Theta \mid y)$ is the posterior likelihood distribution, and $L_{y}(\Theta \mid y)$ is the calculated likelihood distribution of the parameter sets given the set of new observations $(y)$. For example, if the $L_{o}(\Theta)$ and $L_{y}(\Theta \mid y)$ were the likelihood distributions of the previous and posterior wildfire events, respectively, the $L_{p}(\Theta \mid y)$ would be the likelihood distribution obtained from updating the previous likelihood with the information gathered for the posterior wildfire. We used two distinct approaches that mainly differed on the composition of the 'new data' used to update the prior likelihood values.

For the first approach, the posterior likelihoods of a given case study were estimated by combining the likelihoods of all the remaining case studies. This allowed us to evaluate the level 
of applicability of the parameter sets calibrated for a specific case study when applied to other case studies, hereafter referred to as the leave-one-out likelihood approach. In the second approach, the posterior likelihoods were estimated by considering the information obtained from wildfires that had occurred previously. The case studies were ordered based on their occurrence date and the posterior likelihoods of the parameter sets for a given case study were estimated by combining the likelihoods of all past case studies, hereafter referred to as the iterative likelihood approach.

To evaluate the degree of improvement in the predictions and the uncertainty reduction caused by integrating additional data, we analyzed the $S p D$ temporal distribution of: i) the reference simulation; ii) the simulations using the iterative likelihood considering all the previous case studies; and iii) only the first case study (hereafter initial likelihood). This analysis was only performed for the most recent case study (COV).

We also compared the impact of the uncertainty arising from the major input variables and the ROS adjustment factors on the prediction accuracy. This was done by comparing the $S p D_{\text {ratio }}$ distribution derived from propagating uncertainty in the major input variables and the ROS adjustment factors, for each case study independently. For the ROS adjustment factors we used the leave-one-out likelihood approach. The analysis was done for all the case studies.

\section{Results}

\subsection{Impact of ROS adjustment factor uncertainty on prediction accuracy}

Applying the ROS adjustment factors using the weights given by the leave-one-out likelihood approach leads to a general improvement in prediction accuracy (Fig. 1). This is shown by the consistent decrease in $S p D_{\text {ratio }}$, i.e. a decrease in spatial discrepancy when compared with the reference simulations that had no adjustment. The median $S p D_{\text {ratio }}$ is below $-25 \%$ for all case studies, with some fires presenting substantial declines (e.g. CBR2 and LL). Additionally, the interquartile range remains below $0 \%$, showing a consistent decrease in the satellite-simulation discrepancy. This consistent improvement in prediction accuracy is minor for some case studies, 
such as CBR1, MCQ1 and MCQ3, where the $S p D_{\text {ratio }}$ does not drop below $-50 \%$, but is 326 substantial in other case studies, such as CBR2 and LL (also seen in Fig. 1).

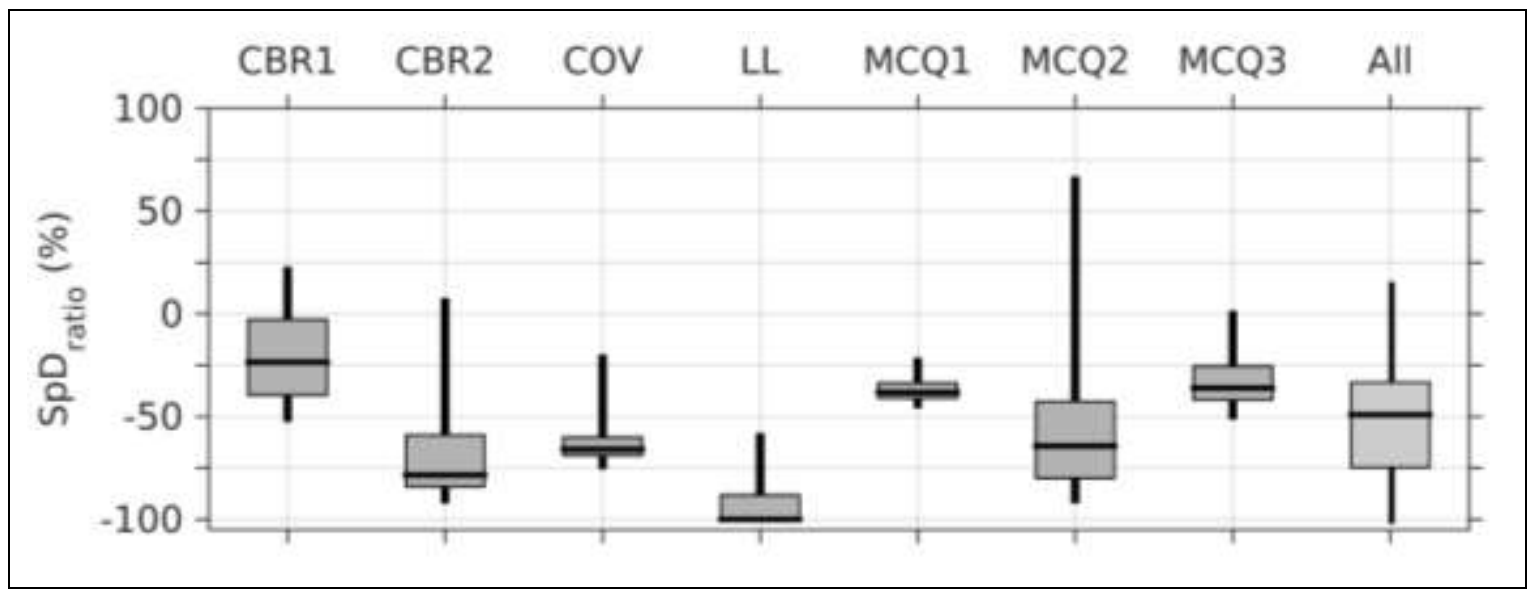

Fig. 1. Distribution of $S p D_{\text {ratio }}$ for the case studies using the ROS adjustment factors tuned for all the remaining case studies (box and whisker plots). For each case study, the gray box represents the interquartile range, the thick horizontal line the median, and the vertical thick lines the 5th and 95th percentiles of the $S p D_{\text {ratio. }}$ Each distribution comprises the median $S p D_{\text {ratio }}$ weighted by the leave-one-out posterior likelihoods 'All' encompasses all case studies.

The update of the posterior likelihoods based on the information collected for past wildfires 328 (i.e. the iterative likelihood approach) leads to an overall improvement of prediction accuracy 329 (Fig. 2). Once again, the median $S p D_{\text {ratio }}$ is below $-25 \%$ for all case studies and the interquartile 330 range was always below $0 \%$. The consistent decrease in the satellite-simulation discrepancy is minor in some case studies, such as CBR1, MCQ1 and MCQ3, but is substantial for others, such as the more recent case studies, LL and COV, which burned in 2004 and 2005, respectively. 


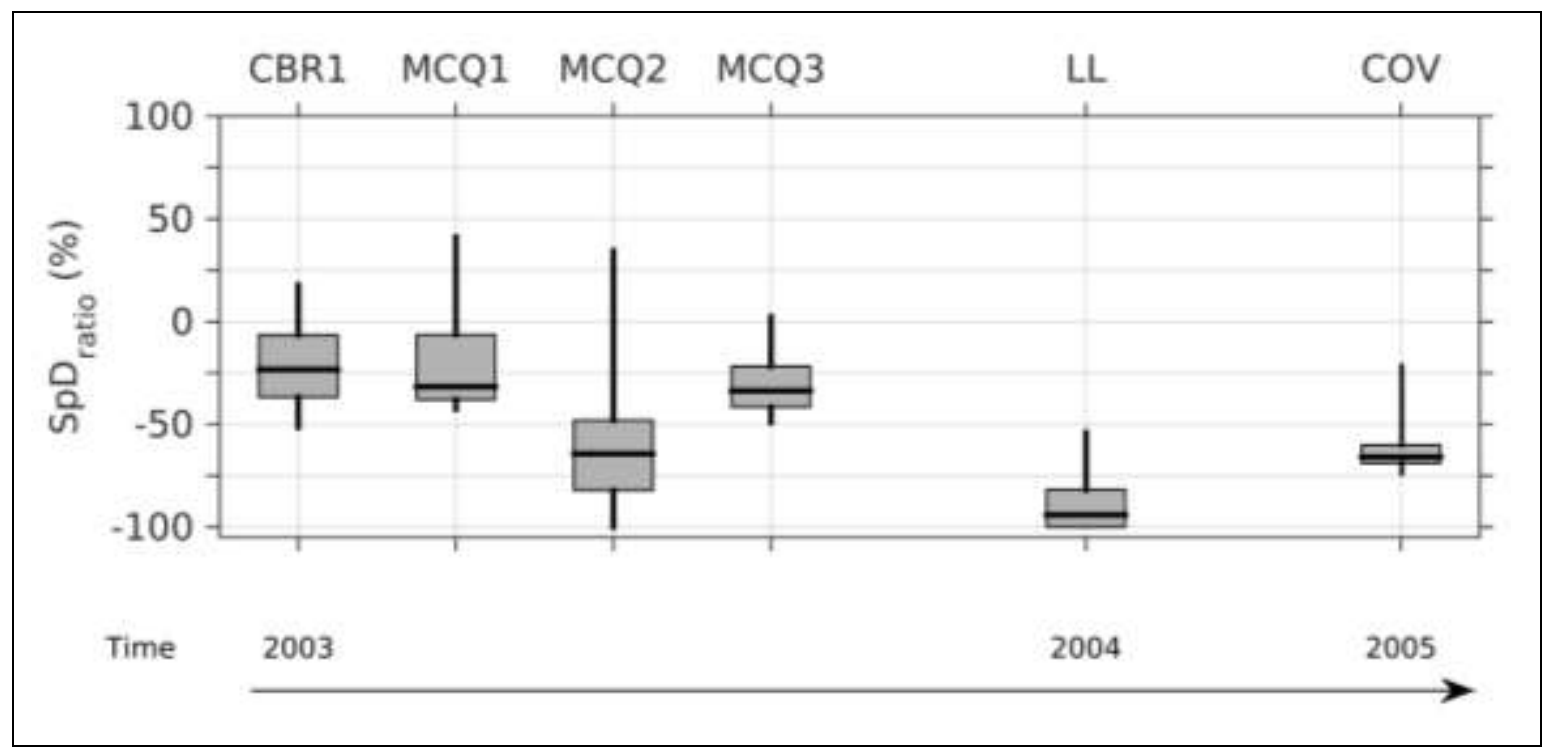

Fig. 2. Distribution of the $S p D_{\text {ratio }}$ for the case studies using the ROS adjustment factors tuned for the case studies that occurred previously. For each case study, the gray box represents the interquartile range, the thick horizontal line the median, and the vertical thick lines the $5^{\text {th }}$ and $95^{\text {th }}$ percentiles of the $\mathrm{SpD}_{\text {ratio }}$. Each distribution comprises the median $S p D_{\text {ratio }}$ weighted by the iterative posterior likelihoods

The distribution of the $S p D$ along elapsed time for the most recent case study (COV) shows a pronounced decrease in the $S p D$ from the reference simulation to the initial likelihood simulations, but a hardly noticeable decrease from the latter to the posterior likelihood

336 simulations (Fig. 3). The median $S p D$ is ca. $1 \mathrm{~km}$ up to about $35 \mathrm{~h}$ of elapsed time for all 337 simulations, and afterwards the median $S p D$ increases pronouncedly for the reference simulation 338 (over $6 \mathrm{~km}$ ) accompanied by a minor increase in the initial and posterior likelihood simulations 339 (ca. $2.5 \mathrm{~km}$ ). The variability of the $S p D$ (represented by the $90 \%$ interval) decreases when using 340 data from new case studies, i.e. from initial to posterior likelihood simulations, suggesting that 341 prediction uncertainty decreases with additional data. 


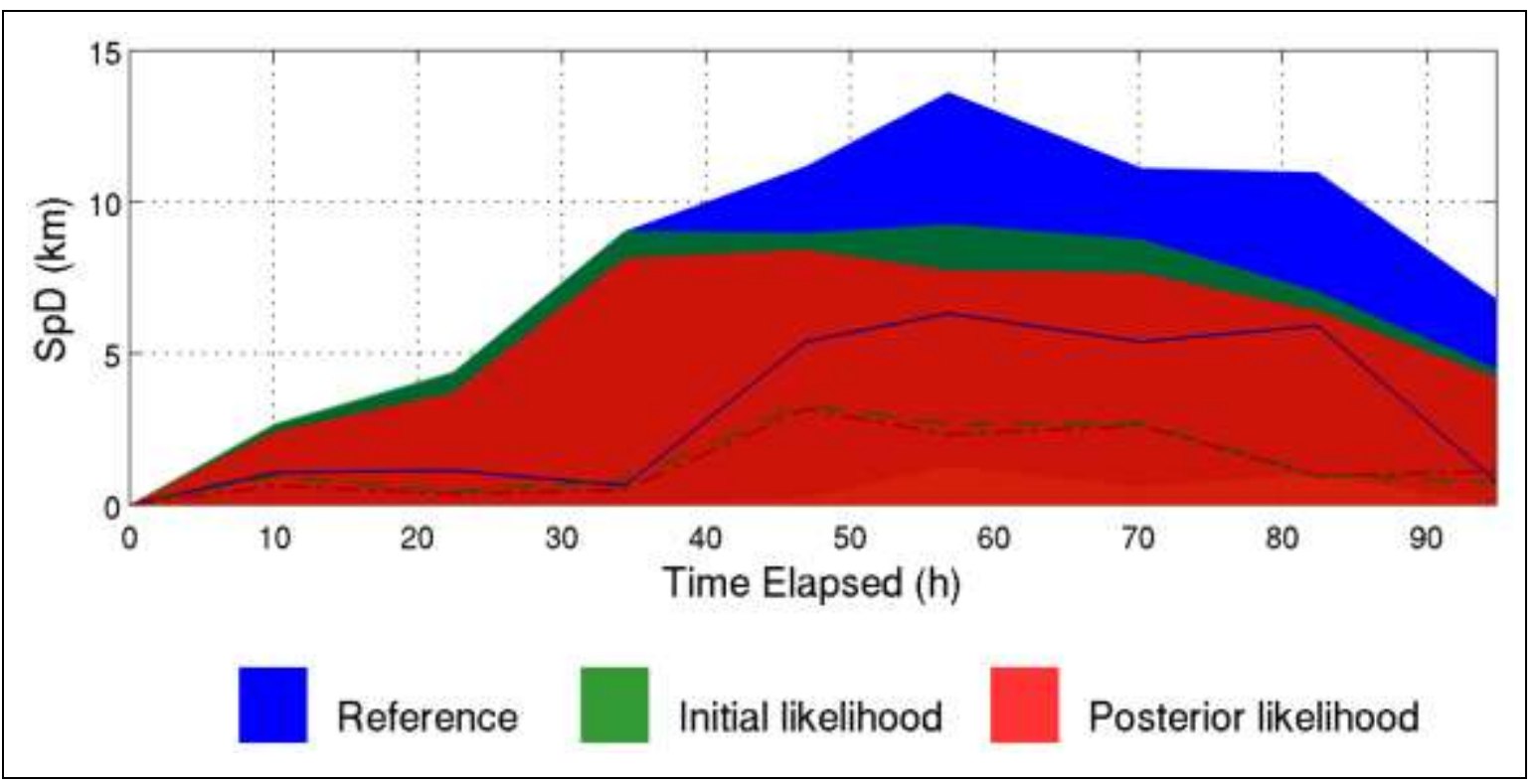

Fig. 3. Temporal distribution of the $S p D$ for the COV case study, considering the reference simulation (blue), the initial (green) and the posterior likelihood (red) simulations. The areas show the $90 \%$ uncertainty limits and the lines refer to the median values over elapsed simulation time.

\subsection{Relation with the major input variables}

The uncertainty in the major input variables and in the ROS adjustment factors has widely variable and heterogeneous impacts on prediction accuracy depending on the case study (Fig. 4). The most obvious distinction is related with the magnitude of the impact of uncertainty on prediction accuracy, represented by the variability of the $S p D_{\text {ratio }} 90 \%$ interval. The impact is very small for CBR1, MCQ1 and MCQ3, contrasting with the remaining case studies. These three case studies are discussed separately below. For the remaining, CBR2 and LL correspond to elongated-shape wildfires, for which the uncertainty in wind speed and direction has a large impact on the $S p D_{\text {ratio }}$, often increasing the value of $S p D_{\text {ratio }}$ although with large variability among all runs. The COV and MCQ2 wildfires have complex burned area perimeters and uncertainty in fuel model assignment leads to a widely variable $S p D_{\text {ratio }}$ response, with significant prediction accuracy improvement and decrease, respectively. Considering the latter

356 four case studies, the uncertainty in ignition location has a relevant impact on $S p D_{\text {ratio }}$ for the 357 case studies with multiple (and distinct) ignition areas, such as LL and MCQ2 (see Sá et al. 2017 358 for details). 
Despite all the above mentioned patterns, reducing the uncertainty in the ROS adjustment 360 factors leads to an overall decrease in the $S p D_{\text {ratio }}$ that is consistently larger than the one resulting 361 from propagating the uncertainty in any of the major input variables.

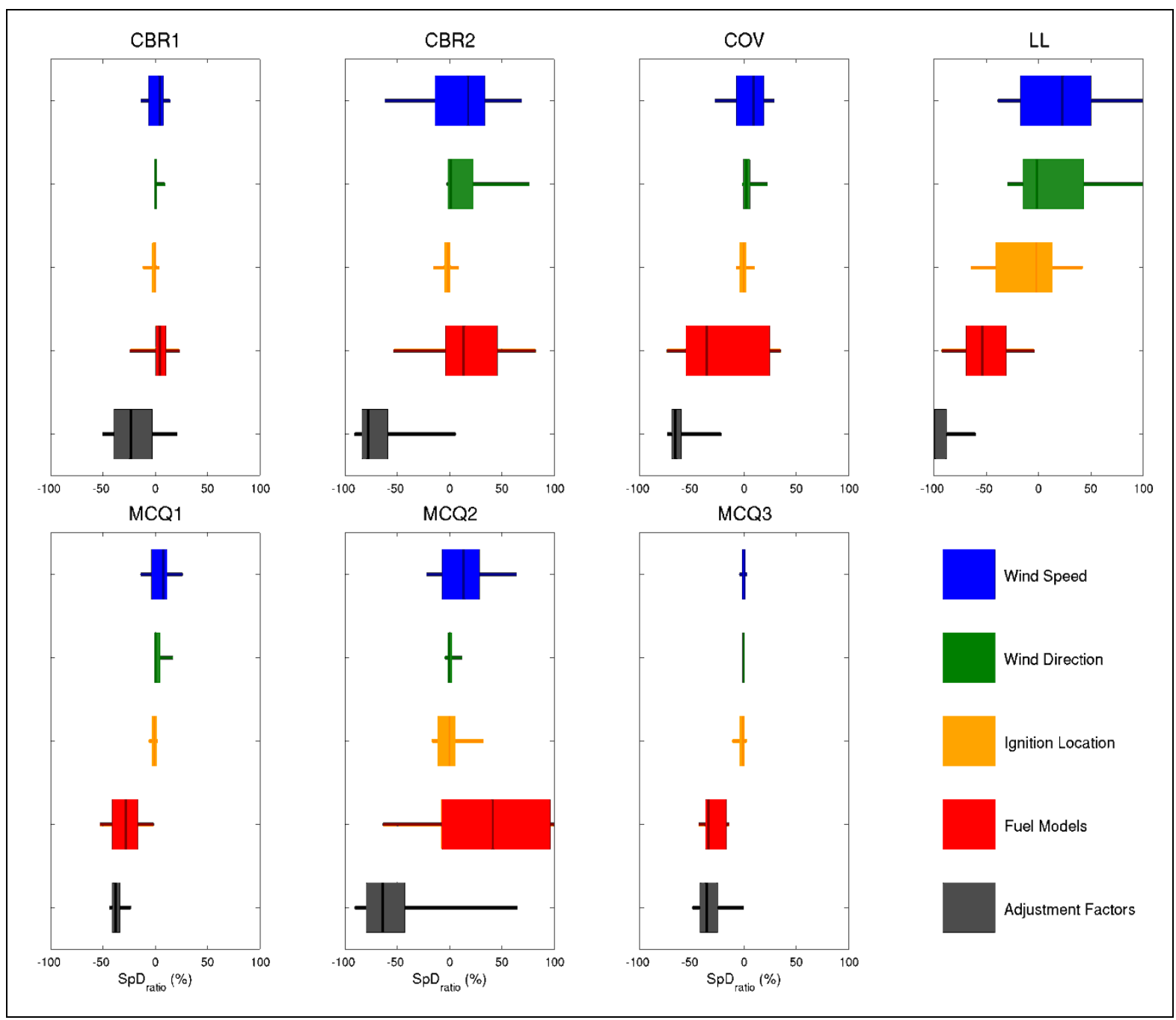

Fig. 4. The impact of the uncertainty in the major input variables and the ROS adjustment factors on the $S p D_{\text {ratio }}$, for each case study individually.

\subsection{Implications for fire management}

The reference simulations show a consistent underestimation of fire spread when compared with the final mapped burned area perimeter (Fig. 5), as stated by Benali et al. (2016a) and Sá et al. (2017). Applying the leave-one-out likelihoods to calculate the burn probability maps leads to 
366 a decrease in fire spread underestimation. The area deemed as "probable" is always larger than 367 the reference simulation, and the one deemed as "very probable" is still larger, except for the 368 CBR1 case study. The CBR1, MCQ1 and MCQ3 have considerable parts of their burned areas 369 with "null probability" class of fire spread. For the remaining four case studies, the burned areas 370 are entirely or almost entirely covered with "medium probability" to "very probable" classes 371 (Fig. 5).

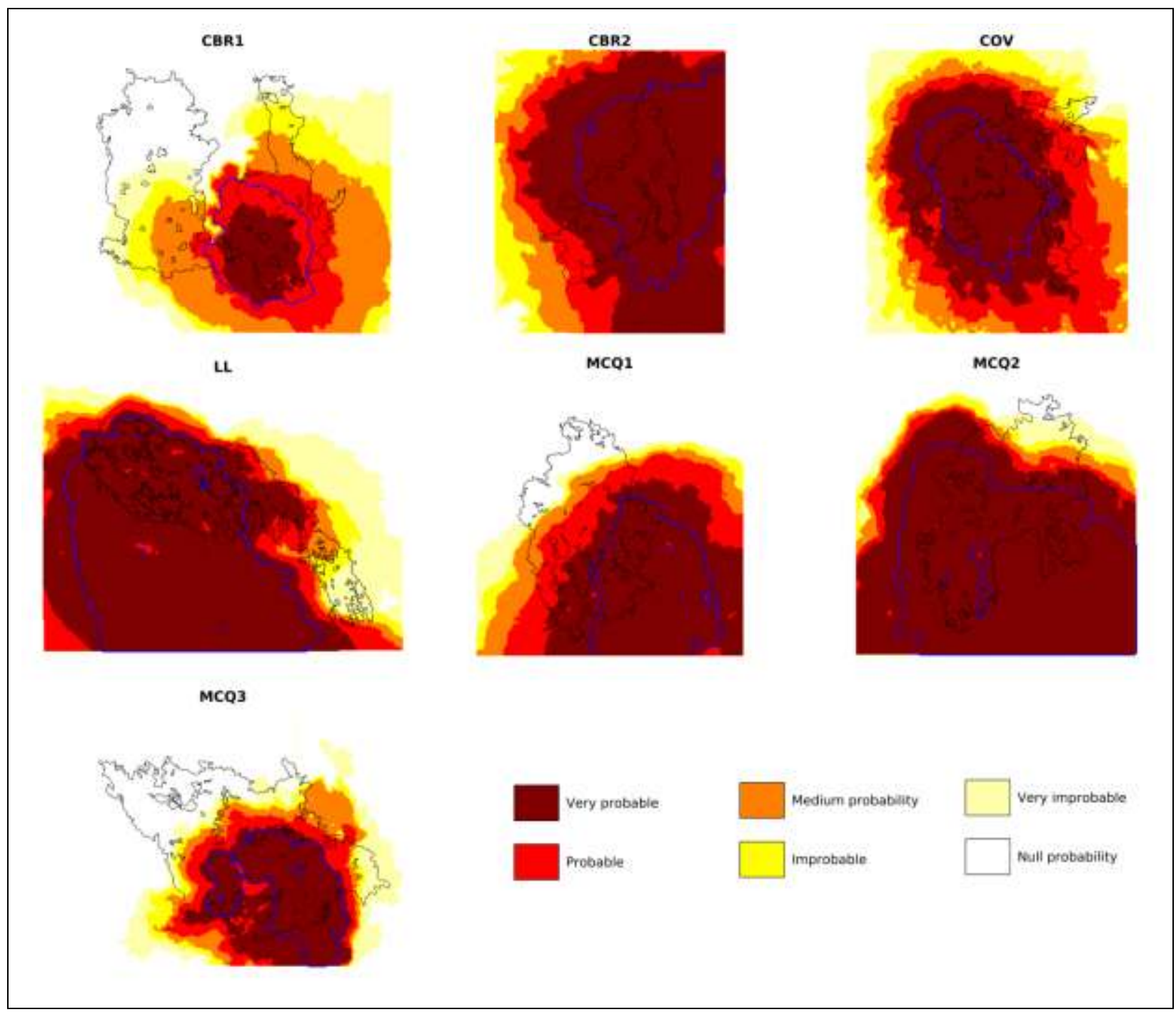

Fig. 5. Burn probability maps based on leave-one-out likelihood. The observed burned area perimeter is mapped in black line and the burned area perimeter resulting from the reference simulation (i.e. no adjustment) is mapped in blue line. 
Integrating the ROS adjustment factor uncertainty consistently increases the probability of

373 burning inside the burned area perimeter, when compared with the integration of both fuel model 374 assignment and wind speed uncertainty (Fig. 6). However, it also largely increases the simulated 375 burn probability outside the mapped fire perimeter. For some case studies, the median increase in 376 non-burned areas is larger than the median increase in burned areas (e.g. COV; CBR2), while for 377 other case studies it is not (e.g. MCQ1).

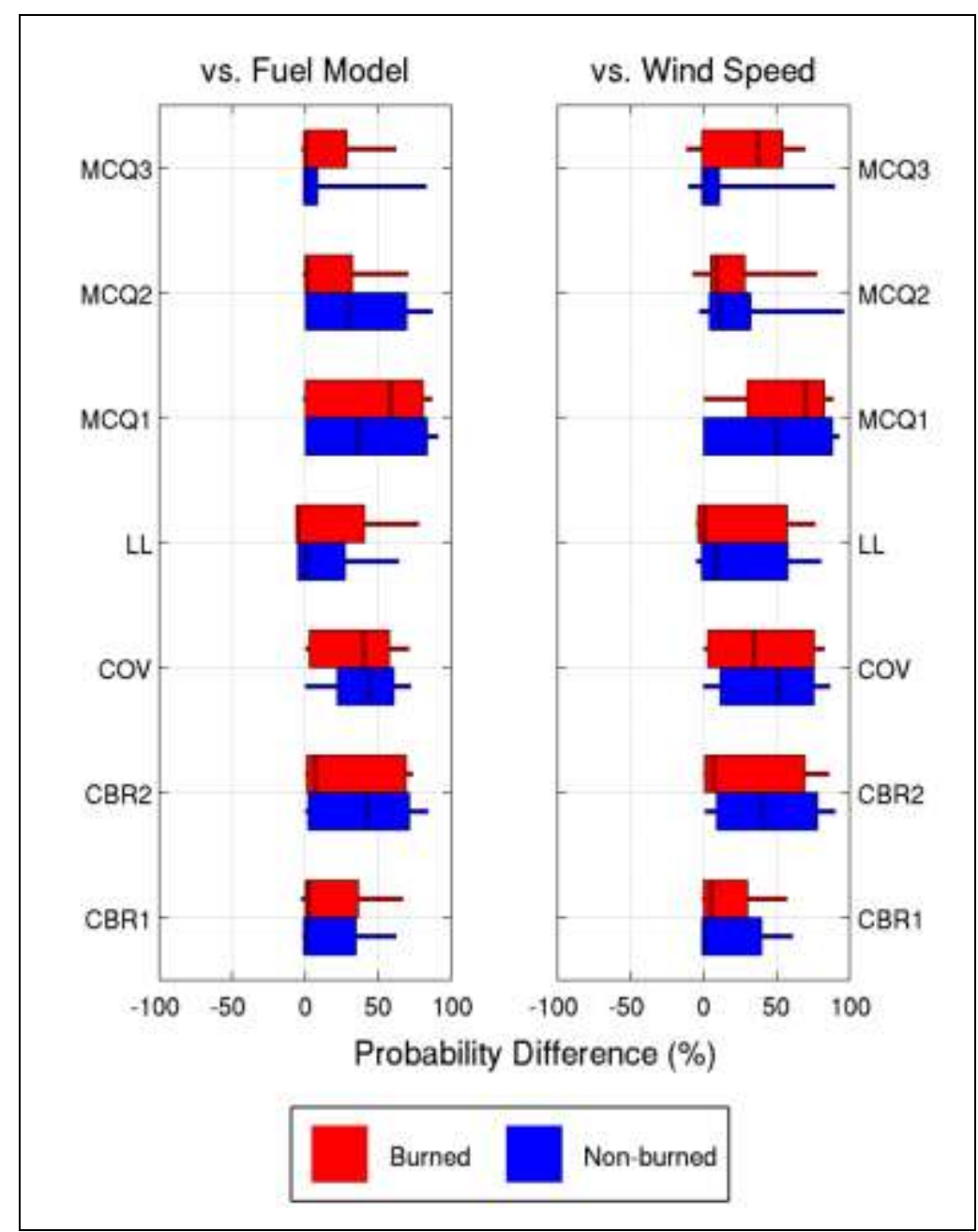

Fig. 6. Difference between the burn probability accounting for the uncertainty in ROS adjustment factors versus the burn probability accounting for the fuel model assignment (left) and the wind speed uncertainty (right). The distribution of the differences is calculated inside (red) and outside (blue) the burned area perimeter. 


\section{Discussion}

\subsection{Understanding the role of the ROS adjustment factors}

Our results show that the accuracy of fire spread predictions can be improved by integrating information from past large wildfires. Furthermore, the most likely parameter sets are applicable to multiple case studies and improve the corresponding fire spread predictions. Improving the accuracy of fire spread simulations can significantly increase their reliability, potentially contributing to support critical fire management decisions. This is particularly relevant, and can be a cost-effective alternative in a resource-limited context, where improving the quality of the input variables and/or modeling systems can be unfeasible in the short-term.

The impact of uncertainty of input variables on the prediction accuracy varies markedly between case studies. For example, counter intuitively, for most case studies integrating uncertainty in wind data barely contributes towards improving simulation results. For some case studies, the impact of data uncertainty on improving prediction accuracy is small, suggesting that improvements on input data quality would not be effective (e.g. MCQ1, 2 and 3). Regardless, the ROS adjustment factors uncertainty strongly counterbalances the negative impacts of input variable uncertainty on prediction accuracy. This clearly shows that, without additional information or significant improvements on the quality of the major input variables, the negative impacts of data uncertainty can be compensated and 'swept under the rug', leading to more reliable fire spread predictions.

Although the ROS adjustment factors are used only to tune the estimated ROS for each individual fuel model, these parameters can affect the simulated fire patterns considerably. Tuning such parameters compensates for prediction errors, not only associated with the incorrect parameterization and/or assignment of fuel models, but also with the remaining input variables. In practice, these parameters also counterbalance, at least partially, the uncertainties resulting for example from model applicability, knowledge limitations, model structure and natural variability (Albini 1976; Alexander and Cruz 2013b; Cruz 2010). Unfortunately, in this context, the most likely parameter sets cannot be used to identify fuel models needing improvement. In fact, the 
large variability and strong compensation between the most likely parameters are strong indicators of the existence of multiple acceptable system simulators, i.e. equifinality.

The use of likelihood weights to describe the uncertainty in the ROS adjustment factors increased the estimated burn probability for all case studies. To compensate for the underestimation of fire spread in the reference simulations (Benali et al. 2016a; Sá et al. 2017), the most likely parameter sets increased the estimated ROS (i.e. adjustment factors above one, not shown). Other studies have documented the underprediction of fire spread rates (Cruz and Alexander 2013) and specifically using FARSITE (Arca et al. 2007). As a consequence, the estimated burn probability increased both for burned and non-burned areas, and for some cases, it was larger in the latter. While we acknowledge that increasing the probability of non-burned areas can be considered a downside of the methodology and can have important implications for fire management, it must be considered that these results were obtained without simulating fire suppression efforts. Moreover, because of its potential consequences, it is fire spread underestimation that represents a larger concern in an operational context (Cheney, 1981).

For the CBR1, MCQ1 and MCQ3 case studies, the uncertainty in both input variables and ROS adjustment factors had a smaller impact on prediction accuracy when compared with the remaining cases. These case studies had considerable parts of their burned areas covered by "null probability" estimates, shown both by the burned area perimeter and satellite active fire data. These results suggest model structural errors and/or epistemic errors, superimposing the uncertainty in input variables and ROS adjustment factors. For instance, spotting was not simulated and most likely occurred in some of the above mentioned case studies, since it is a common feature in Portuguese large wildfires (http://www.fire.uni-freiburg.de/iffn/iffn_34/03IFFN-34-Portugal-Country-Report-2.pdf). According to the Portuguese Rural Fire Database, the CBR1 wildfire had multiple ignitions dispersed over space and time, which were not simulated (http://www.icnf.pt/portal/florestas/dfci/inc/estat-sgif). The MCQ1 wildfire had a strong shift in the prevailing winds, from Southeast to North, which transformed the southern flank into a large burning fire front. After careful inspection, these complex patterns were not correctly reproduced by the input wind data, possibly due to epistemic errors. Although the estimated likelihoods integrate several sources of error and a great deal of uncertainty is encompassed in the ROS adjustment factors, it should not be expected to compensate strong limitations of the modeling 
435

436

437

438

system. Whether the low impact on the variability of prediction accuracy is indicative of strong model structural errors requires further investigation. Nevertheless, identifying and understanding why models fail are crucial steps towards their improvement (Beven and Binley 2014).

\subsection{Limitations}

A thorough investigation of the factors behind the inability to accurately predict the fire spread of the historical case studies is beyond the scope of the work. The impact of input data uncertainties on the accuracy of fire spread predictions has been addressed in a preceding work (Benali et al. 2016a). As mentioned, the fact that spotting fires were not simulated may be one of the major causes behind the fact that some case studies had considerable parts of their burned area without any estimated fire spread. Nevertheless, although we recognize that data quality and model settings need to be improved, the results clearly show a relative improvement in the prediction accuracy by calibrating the ROS adjustment factors. On the other hand, it cannot be expected that parametric uncertainty can encompass all the uncertainties resulting from inadequate or an incomplete model structure, as clearly mentioned by Beven and Binley (1992).

We acknowledge that satellite active fire data also have errors and only provide a snapshot of the observed fire spread. Previous studies thoroughly discussed the role and limitations of satellite data to monitor fire progression and evaluate fire spread simulations (Coen and Schroeder 2013; Hawbaker et al. 2008; Sá et al. 2017). Nevertheless, we would like to reinforce that satellite active fire data have a large potential to provide important insights on the fire growth patterns of large and long-lasting wildfires, thus providing a robust data source for evaluating and consequently improving fire spread simulations.

Other limitations were related with the calibration of the ROS adjustment factors. The spatial variability and the accuracy of the fuel maps inevitably influence the estimated likelihoods for each parameters set. The methodology has limited applicability for wildfires that had a significant contribution of crown and/or spot fires, since the ROS adjustment factors refer only to surface rate of spread. However, the GLUE methodology can be extended to other parameters that control the behavior of spotting and/or crown fires. 
Finally, we acknowledge that the formal definition of an appropriate likelihood function, which is essential to estimate predictive uncertainty under the GLUE methodology, is inherently subjective as stressed by Beven and Binley (1992). The initial parameter distribution is also subjective, although the Uniform distribution has been recognized as the most appropriate in situations with lack of a priori knowledge about the parameter distributions and, the priors become increasingly less important with the addition of new data (Beven and Binley 2014). The definition of the likelihood function should be carefully evaluated by the modeler, by assessing intermediate results, to assess the suitability of the measure for the desired purpose. We have addressed these issues, but acknowledge that other likelihood function could be equally or even more adequate than the one proposed.

\subsection{Future Applications}

Using information from previous wildfires and updating the correspondent likelihoods improved prediction accuracy and reduced $S p D$ variability for the posterior wildfires. This important result suggests that integrating additional fire data can reduce predictive uncertainty, as stated by Beven and Binley (1992), making the information provided to fire managers more reliable. It is expected that with additional case studies the posterior likelihoods greater than zero will become increasingly well constrained. This issue requires further analysis by integrating additional wildfires and using more sophisticated measures to assess the impact on predictive uncertainty (Beven and Binley 2014).

The modifications to compute the $S p D$ index improved its suitability to evaluate the quality of fire spread predictions, focusing on the description of the broad spatio-temporal patterns of fire spread and on the flaming front. The errors and limitations associated with satellite active fire data have been thoroughly described in previous studies (Benali et al. 2016a; Benali et al. 2016b; Hawbaker et al. 2008). We acknowledge that the satellite active fire data are imperfect, and it should not be expected fire spread predictions to be better than the former. These epistemic errors will be difficult to separate but should be, at least partially, contained in the estimation prediction bounds. Future improvements in $S p D$ calculation, including the use of other satellite or airborne thermal data, will certainly improve the determination of the most likely parameter sets. In this context, the Visible Infrared Imaging Radiometer Suite (VIIRS), 
492 with improved spatial resolution, has a large potential to provide relevant high quality 493 information regarding the spread patterns of wildfires (Schroeder et al. 2014).

494

495

496

497

498

499

500

501

502

503

504

505

506

507

508

509

510

511

512

513

514

515

516

517

518

519

The application of other fire spread evaluation measures (e.g. Cui and Perera 2010; Duff et al. 2013; Filippi et al. 2014; Fujioka 2002) should be feasible as long as they i) provide a measure of goodness-of-fit in a general sense, ii) are monotonic and continuous, and iii) the likelihood function is carefully adapted (Beven and Binley 1992). A complete discussion regarding the GLUE methodology and the importance of correctly defining the likelihood function is provided by Beven and Binley (2014).

The posterior likelihood distribution calculated for previous case studies (iterative likelihood approach) can be used to project the predictive uncertainty for new wildfires and has great potential to be applied to future wildfires under an operational context. Additionally, the approach presented can provide complementary information that can be useful for fire management, such as error bounds and probabilistic outcomes (Cruz 2010). The GLUE methodology has the flexibility to be applied to new wildfires using different sets of input variables and/or model settings. These different settings should be mirrored in the posterior likelihoods. Nevertheless, further work is still needed by applying the methodology to a larger number of case studies that cover a wide range of wildfire characteristics regarding, among others: i) size, including smaller, more frequent and lower intensity wildfires; ii) growth rate, including slow and fast burning wildfires; and iii) type, including surface and crown fires. Additionally, further work should integrate multiple model structures towards a more complete definition of fire spread uncertainty.

\section{Conclusions}

Improving fire spread predictions for management purposes, by using higher quality input data or enhanced models, can be expensive, unfeasible and even impossible under the current scientific paradigm. Within this context, we showed that fire spread predictions can be improved by appropriately defining the distribution of the 'tunable' parameters contained in the fire spread modeling system. Of special importance, we showed that fire spread predictions can be continuously improved by 'learning' from past wildfires. The uncertainty contained in the major 
520 input variables (wind speed and direction, ignition location and fuel models) can be 'swept under 521 the rug' to a certain extent, and their negative impacts can be counterbalanced through the use of 522 more appropriate parameter sets.

523 The framework proposed can be applied to future wildfires and the posterior likelihoods can 524 be continuously updated by including new observations. Arguably, it has a large potential to 525 improve future fire spread predictions, improving their reliability and usefulness to support fire 526 management and decision making processes, thus potentially reducing the negative impacts of 527 wildfires.

528 6. Acknowledgements

529 This study was supported by the FIRE-MODSAT project (EXPL/AGR-FOR/0488/2013) 530 funded by the Fundação para a Ciência e a Tecnologia. The Centro Estudos Florestais was 531 financed by the latter entity under the grant UID/AGR/00239/2013. We thank the i) MAR 532 research group of the University of Murcia for kindly providing the weather data, ii) John Benoit 533 from the USDA Forest Service for assistance with the FARSITE command line version; and iii) 534 Renata Pinto (University of Lisbon) for processing part of the input data. 


\section{References}

Ager, A.A., Vaillant, N.M., \& Finney, M.A. (2010). A comparison of landscape fuel treatment strategies to mitigate wildland fire risk in the urban interface and preserve old forest structure. Forest Ecology and Management, 259, 1556-1570

Albini, F.A. (1976). Estimating wildfire behavior and effects. USDA For. Serv., Intermt. For. Range Exp. Stn., Ogden, UT. Gen. Tech. Rep. INT-30. 92 p.

Alexander, M.E., \& Cruz, M.G. (2013a). Are the applications of wildland fire behaviour models getting ahead of their evaluation again? Environmental Modelling \& Software, 41, 65-71

Alexander, M.E., \& Cruz, M.G. (2013b). Limitations on the accuracy of model predictions of wildland fire behaviour: A state-of-the-knowledge overview. The Forestry Chronicle, 89, 372-383

Anderson, H.E. (1982). Aids to determining fuel models for estimating fire behavior. The Bark Beetles, Fuels, and Fire Bibliography, 143

Anderson, K., Reuter, G., \& Flannigan, M.D. (2007). Fire-growth modelling using meteorological data with random and systematic perturbations. International Journal of Wildland Fire, 16, 174

Anderson, K.R., Englefield, P., Little, J.M., \& Reuter, G. (2009). An approach to operational forest fire growth predictions for Canada. International Journal of Wildland Fire, 18, 893

Arca, B., Duce, P., Laconi, M., Pellizzaro, G., Salis, M., \& Spano, D. (2007). Evaluation of FARSITE simulator in Mediterranean maquis. International Journal of Wildland Fire, 16, 563

Ascoli, D., Vacchiano, G., Motta, R., \& Bovio, G. (2015). Building Rothermel fire behaviour fuel models by genetic algorithm optimisation. International Journal of Wildland Fire, 24, 317-328

Bachmann, A., \& Allgöwer, B. (2002). Uncertainty propagation in wildland fire behaviour modelling. International Journal of Geographical Information Science, 16, 115-127

Benali, A., Ervilha, A.R., Sá, A.C.L., Fernandes, P.M., Pinto, R.M.S., Trigo, R.M., \& Pereira, J.M.C. (2016a). Deciphering the impact of uncertainty on the accuracy of large wildfire spread simulations. Science of the total environment, 569, 73-85

Benali, A., Russo, A., Sá, A.C., Pinto, R., Price, O., Koutsias, N., \& Pereira, J. (2016b). Determining Fire Dates and Locating Ignition Points With Satellite Data. Remote Sensing, 8, 326

Beven, K., \& Binley, A. (1992). The future of distributed models: model calibration and uncertainty prediction. Hydrological processes, 6, 279-298

Beven, K., \& Binley, A. (2014). GLUE: 20 years on. Hydrological processes, 28, 5897-5918

Bossard, M., Feranec, J., \& Otahel, J. (2000). CORINE land cover technical guide: Addendum 2000. In: European Environment Agency Copenhagen

Cai, L., He, H.S., Wu, Z., Lewis, B.L., \& Liang, Y. (2014). Development of standard fuel models in boreal forests of northeast China through calibration and validation. PLoS One, 9, e94043

Calkin, D.E., Thompson, M.P., Finney, M.A., \& Hyde, K.D. (2011). A Real-Time Risk Assessment Tool Supporting Wildland Fire Decisionmaking. Journal of Forestry, 274-280

Coen, J.L., \& Schroeder, W. (2013). Use of spatially refined satellite remote sensing fire detection data to initialize and evaluate coupled weather-wildfire growth model simulations. Geophysical Research Letters, 40, 5536-5541

Cruz, M.G. (2010). Monte Carlo-based ensemble method for prediction of grassland fire spread. International Journal of Wildland Fire, 19, 521

Cruz, M.G., \& Alexander, M.E. (2010). Assessing crown fire potential in coniferous forests of western North America: a critique of current approaches and recent simulation studies. International Journal of Wildland Fire, 19, 377

Cruz, M.G., \& Alexander, M.E. (2013). Uncertainty associated with model predictions of surface and crown fire rates of spread. Environmental Modelling \& Software, 47, 16-28

Cruz, M.G., \& Fernandes, P.M. (2008). Development of fuel models for fire behaviour prediction in maritime pine (Pinus pinaster Ait.) stands. International Journal of Wildland Fire, 17, 194-204

Cui, W., \& Perera, A. (2010). Quantifying spatio-temporal errors in forest fire spread modelling explicitly. Journal of Environmental Informatics, 16, 19-26 
DiMiceli, C., Carroll, M., Sohlberg, R., Huang, C., Hansen, M., \& Townshend, J. (2011). Annual Global Automated MODIS Vegetation Continuous Fields (MOD44B) at $250 \mathrm{~m}$ Spatial Resolution for Data Years Beginning Day 65, 2000-2010, Collection 5 Percent Tree Cover. University of Maryland, College Park

Duff, T.J., Chong, D.M., \& Tolhurst, K.G. (2013). Quantifying spatio-temporal differences between fire shapes: Estimating fire travel paths for the improvement of dynamic spread models. Environmental Modelling \& Software, 46, 33-43

Duguy, B., Alloza, J.A., Röder, A., Vallejo, R., \& Pastor, F. (2007). Modelling the effects of landscape fuel treatments on fire growth and behaviour in a Mediterranean landscape (eastern Spain). International Journal of Wildland Fire, 16, 619

Ervilha, A., Pereira, J., \& Pereira, J. (2017). On the parametric uncertainty quantification of the Rothermel's rate of spread model. Applied Mathematical Modelling, 41, 37-53

Farr, T.G., Rosen, P.A., Caro, E., Crippen, R., Duren, R., Hensley, S., Kobrick, M., Paller, M., Rodriguez, E., \& Roth, L. (2007). The shuttle radar topography mission. Reviews of geophysics, 45

Fernandes, P. (2005). Equivalência genérica entre os modelos de combustível do USDA Forest Service (Anderson, 1982) e as formações florestais portuguesas. In: Guia metodológico para elaboração do Plano Municipa/Intermunicipal de Defesa da Floresta Contra Incêndios In: Direção Geral dos Recursos Florestais

Filippi, J.-B., Mallet, V., \& Nader, B. (2014). Representation and evaluation of wildfire propagation simulations. International Journal of Wildland Fire, 23, 46-57

Finney, M.A. (2004). FARSITE: Fire Area Simulator-Model Development and Evaluation. USDA Research Paper RMRS-RP-4

Finney, M.A., Grenfell, I.C., McHugh, C.W., Seli, R.C., Trethewey, D., Stratton, R.D., \& Brittain, S. (2011). A Method for Ensemble Wildland Fire Simulation. Environmental Modeling \& Assessment, $16,153-167$

Fujioka, F.M. (2002). A new method for the analysis of fire spread modeling errors. International Journal of Wildland Fire, 11, 193-203

Giglio, L., Descloitres, J., Justice, C.O., \& Kaufman, Y.J. (2003). An Enhanced Contextual Fire Detection Algorithm for MODIS. Remote Sensing of Environment, 87, 273-282

Hawbaker, T.J., Radeloff, V.C., Syphard, A.D., Zhu, Z., \& Stewart, Z.I. (2008). Detection rates of the MODIS active fire product in the United States. Remote Sensing of Environment, 112, 2656-2664

Hilton, J.E., Miller, C., Sullivan, A.L., \& Rucinski, C. (2015). Effects of spatial and temporal variation in environmental conditions on simulation of wildfire spread. Environmental Modelling \& Software, 67, 118-127

Keane, R.E., \& Reeves, M. (2012). Use of Expert Knowledge to Develop Fuel Maps for Wildland Fire Management. In C.A.D. A.H. Perera, and C.J. Johnson (Ed.), Expert knowledge and its application in landscape ecology. (p. 211-228). New York, USA: Springer Science and Business Media, LLC

Kochanski, A.K., Jenkins, M.A., Mandel, J., Beezley, J.D., \& Krueger, S.K. (2013). Real time simulation of 2007 Santa Ana fires. Forest Ecology and Management, 294, 136-149

Liu, Y., Jimenez, E., Hussaini, M.Y., Okten, G., \& Goodrick, S. (2015). Parametric uncertainty quantification in the Rothermel model with randomised quasi-Monte Carlo methods. International Journal of Wildland Fire, 24, 307

Lorente-Plazas, R., Montávez, J., Jerez, S., Gómez-Navarro, J., Jiménez-Guerrero, P., \& Jiménez, P. (2015). A 49 year hindcast of surface winds over the Iberian Peninsula. International Journal of Climatology, 35, 3007-3023

Mandel, J., Kochanski, A.K., Vejmelka, M., \& Beezley, J.D. (2014). Data Assimilation of Satellite Fire Detection in Coupled Atmosphere-Fire Simulation by WRF-SFIRE. arXiv preprint arXiv:1410.6948

Oliveira, S.L.J., Pereira, J.M.C., \& Carreiras, J.M.B. (2012). Fire frequency analysis in Portugal (1975 2005), using Landsat-based burnt area maps. International Journal of Wildland Fire, 21, 48

Papadopoulos, G.D., \& Pavlidou, F.-N. (2011). A Comparative Review on Wildfire Simulators. IEEE Systems Journal, 5, 233-243 
Parks, S.A. (2014). Mapping day-of-burning with coarse-resolution satellite fire-detection data. International Journal of Wildland Fire, 23, 215

Pinto, R.M., Benali, A., Sá, A.C., Fernandes, P.M., Soares, P.M., Cardoso, R.M., Trigo, R.M., \& Pereira, J.M. (2016). Probabilistic fire spread forecast as a management tool in an operational setting. SpringerPlus, 5, 1205

Pollack, H.N. (2005). Uncertain science... uncertain world. Cambridge University Press

Refsgaard, J.C., van der Sluijs, J.P., Højberg, A.L., \& Vanrolleghem, P.A. (2007). Uncertainty in the environmental modelling process - A framework and guidance. Environmental Modelling \& Software, 22, 1543-1556

Rothermel, R.C. (1972). A mathematical model for predicting fire spread in wildland fuels. USDA For. Serv. Res. Pap. INT-115.

Rothermel, R.C. (1991). Predicting behavior and size of crown fires in the northern Rocky Mountains. USDA For. Serv. Res. Pap. INT-438.

Rothermel, R.C., \& Rinehart, G.C. (1983). Field procedures for verification and adjustment of fire behavior predictions. US Department of Agriculture, Forest Service, Intermountain Forest and Range Experiment Station

Sá, A.C.L., Benali, A., Fernandes, P.M., Pinto, R.M.S., Trigo, R.M., Salis, M., Russo, A., Jerez, S., Soares, P.M., Schroeder, W., \& Pereira, J.M.C. (2017). Evaluating fire growth simulations using satellite active fire data. Remote Sensing of Environment, 190, 302-317

Salis, M., Ager, A.A., Arca, B., Finney, M.A., Bacciu, V., Duce, P., \& Spano, D. (2013). Assessing exposure of human and ecological values to wildfire in Sardinia, Italy. International Journal of Wildland Fire, 22, 549

Salis, M., Arca, B., Alcasena, F., Arianoutsou, M., Bacciu, V., Duce, P., Duguy, B., Koutsias, N., Mallinis, G., \& Mitsopoulos, I. (2016). Predicting wildfire spread and behaviour in Mediterranean landscapes. International Journal of Wildland Fire, 25, 1015-1032

Schroeder, W., Oliva, P., Giglio, L., \& Csiszar, I.A. (2014). The New VIIRS 375m active fire detection data product: Algorithm description and initial assessment. Remote Sensing of Environment, 143, 8596

Scott, J.H., \& Burgan, R.E. (2005). Standard fire behavior fuel models: a comprehensive set for use with Rothermel's surface fire spread model. USDA Forest Service Gen. Tech. Rep. RMRS-GTR-153, Rocky Mountain Research Station, Fort Collins, CO., 66

Sneeuwjagt, R.J., \& Peet, G.B. (1985). Forest fire behaviour tables for Western Australia. WA Department Conservation \& Land Management.

Sullivan, A.L. (2009a). Wildland surface fire spread modelling, 1990-2007. 1: Physical and quasiphysical models. International Journal of Wildland Fire, 18, 349-368

Sullivan, A.L. (2009b). Wildland surface fire spread modelling, 1990-2007. 2: Empirical and quasiempirical models. International Journal of Wildland Fire, 18, 369-386

Sullivan, A.L. (2009c). Wildland surface fire spread modelling, 1990-2007. 3: Simulation and mathematical analogue models. International Journal of Wildland Fire, 18, 387-403

Thompson, M.P., \& Calkin, D.E. (2011). Uncertainty and risk in wildland fire management: a review. Journal of Environmental Management, 92, 1895-1909

van Wagner, C.E. (1977). Conditions for the start and spread of crownfire. Canadian Journal of Forest Research, 7, 23-24

Veraverbeke, S., Sedano, F., Hook, S.J., Randerson, J.T., Jin, Y., \& Rogers, B.M. (2014). Mapping the daily progression of large wildland fires using MODIS active fire data. International Journal of Wildland Fire, 23, 655-667

Webley, P., Riley, K., Thompson, M., Patra, A., \& Bursik, M. (2016). Natural Hazard Uncertainty Assessment: Modeling and Decision Support. John Wiley \& Sons 
